\title{
Introduction to International Journal of Testing Special Issue on Equity and Fairness in Testing and Assessment in School Admissions
}

\author{
Guest Editors: Sang Eun Woo, Bart Wille, \& Stephen G. Sireci
}

Across the globe, educational tests are used for admissions decisions to competitive colleges, universities, high schools, and other programs. The high stakes associated with these tests have important consequences for students, and performance on them can determine whether students reach their academic and career aspirations. For this reason, their use is both widespread and contentious. Recently, the debates over the use of standardized tests in college and graduate admissions has increased, due in large part to concerns about score disparities resulting in disparate admissions outcomes. The International Journal of Testing has published many examples of criticisms and research with respect to admissions testing around the world, including in Chile (Ramirez et al., 2020), Israel (Rapp \& Allalouf, 2003), Saudi Arabia (Tsaousis et al., 2018), Sweden (Wiberg \& von Davier, 2017), and the United States (e.g., Talento-Miller, 2008).

In the U.S. and Chile, public outcry against disparate outcomes for certain groups of students have marshalled in changes in admissions testing programs and the policies associated with them (Koljatic et al., 2021). In the U.S., several colleges and universities have suspended the SAT and ACT requirements for their applicants, which generated a number of heated discussions both within and outside academia. The use of the Graduate Record Examinations (GREs) in graduate admissions is also being hotly debated for similar reasons, and a number of graduate programs in the U.S. have opted to remove the GRE requirement from their admission processes, at least for the upcoming admission cycle. Although educational systems are organized in different manners across the globe, these discussions in the U.S. mirror international debates over the use and implementation of school admissions testing systems. For example, in Chile, the country's university selection test has been strongly protested against, on the ground of social justice and educational equality, and the Ministry of Education responded to these protests by requiring the development of new admissions tests.

The issue of test fairness and equity in school admissions warrants rigorous and systematic investigations from multiple vantage points (e.g., legal, economic, psychometric). In this special issue, we aim to move this important dialogue forward from the psychometric perspective. This issue features four empirical papers and one conceptual paper that each uniquely investigate areas in which school admission testing and assessment can (or should) be made more equitable and fair.

The first two papers reveal there are many unresolved issues of validity and fairness associated with non-standardized assessment tools often used in school admissions. Niessen and Neumann investigated validity and gender bias issues in personal statements using a sample of 806 psychology program applicants at a Dutch university. While no evidence was found for gender disparities in the use of agentic language (nor any meaningful association between agentic language use and ratings of those statements), personal statement ratings were also found to be unrelated to first-year GPA and dropout rates. On the other hand, Dalal and colleagues 
conducted a thorough text analysis of more than 30,000 letters of recommendations submitted for over 10,000 graduate school applicants, and found some systematic differences across racial and gender subgroups of applicants in the content of the letters they received. Interestingly, the authors also reported that graduate programs' admission decisions did not appear to be influenced by the letters of recommendation content.

On the issues of standardized testing, Newman and colleagues sought to address one of the most often-asked questions over the past few years: "Should graduate programs continue to require the GRE?" In their thoughtful and comprehensive analysis on the use of the GRE in graduate school admissions, the authors explicate several key issues - namely, validity of the GRE in predicting graduate school success, achievement gap in test scores, the diversityperformance tradeoff formalized through a Pareto-optimization approach, and three distinct notions of fairness (i.e., equality, test equity, performance equity). Based on these foundations, the authors then illustrate that: (a) dropping the GRE would yield suboptimal admission results especially from the performance standpoint (and making it optional does not help much); and (b) placing different values on admission goals (e.g., maximizing performance vs. diversity) can lead to different implications of programs' decisions on whether to require the GRE or not.

Looking further into the future, the last two articles offer some promising directions. Klieger and colleagues illustrate how standardizing letters of recommendation (also referred to as 'third-party evaluations', 'other-reports' and 'observer ratings') to assess graduate school applicants' socioemotional skills (e.g., resilience, teamwork, planning and organization) can lead to improved fairness outcomes such as greater racial, ethnic and gender diversity while simultaneously improving the overall validity of the selection system in predicting graduate school success. Specifically, the authors analyzed data from three dependent samples of graduate school applicants and students on their GRE test scores, undergraduate and graduate grades (including GPAs), self-reported demographic characteristics such as gender, race and ethnicity, and ratings on the dimensions of the Personal Potential Index (PPI). The PPI is an online multirater assessment tool developed as a way of standardizing reference letters to measure graduate school applicants' socioemotional skills.

Arce and Young provide a helpful conceptual framework for evaluating test efficacy within the context of college admission testing programs, to include the students who take the tests as focal points in the validation process. Defining efficacy as "the ability to produce intended outcomes or impacts on individuals," their framework extends the boundaries of how test validation has been traditionally understood and implemented in practice. The efficacy framework places greater emphasis on evaluating (and improving on) consequences of tests on all college applicants, and thus signifies an important step toward a more well-rounded and forward-looking conversation that involves multiple stakeholder perspectives.

Taken together, these five articles meaningfully contribute to the existing body of research on equity and fairness issues in school admissions, and highlight a number of avenues for future empirical, methodological, and theoretical research. It is our sincere hope this special issue serves as a catalyst for more concerted research efforts and change-oriented discussions that bring about greater equity and fairness in school admissions around the globe. 


\section{References}

Koljatic, M., Silva, M., \& Sireci, S. G. (2021). College admission tests and social responsibility. Educational Measurement: Issues and Practice. https://doi.org/10.1111/emip.12425.

Ramirez, A., Koljatic, M., \& Silva, M. (2020). Coaching $\beta$ in admission test performance: A study of group differences. International Journal of Testing, 20(4), 253-273. https://doiorg.silk.library.umass.edu/10.1080/15305058.2020.1786833.

Rapp, J., \& Allalouf, A. (2003). Evaluating cross-lingual equating. International Journal of Testing, 3(2), 101-117. https://doiorg.silk.library.umass.edu/10.1207/S15327574IJT0302_1.

Talento-Miller, E. (2008). Generalizability of GMAT® validity to programs outside the US. International Journal of Testing, 8(2), 127-142. https://doiorg.silk.library.umass.edu/10.1080/15305050802001193

Tsaousis, I., Sideridis, G., \& Al-Saawi, F. (2018). Differential distractor functioning as a method for explaining DIF: The case of a national admissions test in Saudi Arabia. International Journal of Testing, 18(1), 1-26. https://doiorg.silk.library.umass.edu/10.1080/15305058.2017.1345914.

Wiberg, M., \& von Davier, A. A. (2017). Examining the impact of covariates on anchor tests to ascertain quality over time in a college admissions test. International Journal of Testing, 17(2), 105-126. https://doi-org.silk.library.umass.edu/10.1080/15305058.2016.1277357 